\title{
The Herschel-Heterodyne Instrument for the Far-Infrared (HIFI) ${ }^{\star}$
}

Th. de Graauw ${ }^{1,2,3}$, F. P. Helmich ${ }^{1}$, T. G. Phillips ${ }^{4}$, J. Stutzki ${ }^{5}$, E. Caux ${ }^{6,7}$, N. D. Whyborn ${ }^{1,3}$, P. Dieleman ${ }^{1}$, P. R. Roelfsema ${ }^{1}$, H. Aarts ${ }^{1}$, R. Assendorp ${ }^{1}$, R. Bachiller ${ }^{8}$, W. Baechtold ${ }^{21}$, A. Barcia ${ }^{9}$, D. A. Beintema ${ }^{1}$, V. Belitsky ${ }^{10}$, A. O. Benz ${ }^{11}$, R. Bieber ${ }^{5}$, A. Boogert ${ }^{35}$, C. Borys ${ }^{35}$, B. Bumble ${ }^{12}$, P. Caïs ${ }^{13,14}{ }^{14}$ M. Caris ${ }^{15}$, P. Cerulli-Irelli ${ }^{16}$, G. Chattopadhyay ${ }^{4}$, S. Cherednichenko ${ }^{10}$, M. Ciechanowicz ${ }^{15}$, O. Coeur-Joly ${ }^{6,7}$, C. Comito ${ }^{15}$, A. Cros ${ }^{6,7}$, A. de Jonge ${ }^{1}$, G. de Lange ${ }^{1}$, B. Delforges ${ }^{1,19,20}$, Y. Delorme ${ }^{19,20}$, T. den Boggende ${ }^{1}$, J.-M. Desbat ${ }^{13,14}$, C. Diez-González ${ }^{9}$, A. M. Di Giorgio ${ }^{16}$, L. Dubbeldam ${ }^{1}$, K. Edwards ${ }^{1,17}$, M. Eggens ${ }^{1}$, N. Erickson ${ }^{24}$, J. Evers ${ }^{1}$, M. Fich ${ }^{17}$, T. Finn ${ }^{25}$, B. Franke ${ }^{5}$, T. Gaier ${ }^{12}$, C. Gal ${ }^{5}$, J. R. Gao ${ }^{28}$, J.-D. Gallego 9 , S. Gauffre ${ }^{13,14}$, J. J. Gill ${ }^{12}$, S. Glenz ${ }^{5}$, H. Golstein ${ }^{1}$, H. Goulooze ${ }^{1}$, T. Gunsing ${ }^{1}$, R. Güsten ${ }^{15}$, P. Hartogh ${ }^{18}$, W. A. Hatch ${ }^{12}$, R. Higgins ${ }^{1,26}$, E. C. Honingh ${ }^{5}$, R. Huisman ${ }^{1}$, B. D. Jackson ${ }^{1}$, H. Jacobs ${ }^{1}$, K. Jacobs ${ }^{5}$, C. Jarchow ${ }^{18}$, H. Javadi ${ }^{12}$, W. Jellema ${ }^{1}$, M. Justen ${ }^{5}$, A. Karpov ${ }^{4}$,

C. Kasemann ${ }^{15}$, J. Kawamura ${ }^{12}$, G. Keizer ${ }^{1}$, D. Kester ${ }^{1}$, T. M. Klapwijk ${ }^{28}$, Th. Klein ${ }^{15}$, E. Kollberg ${ }^{10}$, J. Kooi ${ }^{4}$, P.-P. Kooiman ${ }^{1}$, B. Kopf ${ }^{5}$, M. Krause ${ }^{5}$, J.-M. Krieg ${ }^{19,20}$, C. Kramer ${ }^{5}$, B. Kruizenga ${ }^{27}$, T. Kuhnn ${ }^{5}$, W. Laauwen ${ }^{1}$, R. Lai ${ }^{29}$,

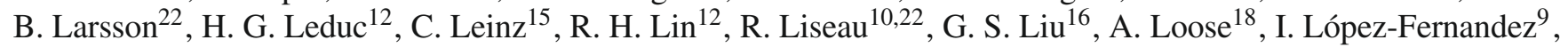
S. Lord ${ }^{35}$, W. Luinge ${ }^{1}$, A. Marston ${ }^{1,33}$, J. Martín-Pintado ${ }^{30}$, A. Maestrini1 ${ }^{12}$, F. W. Maiwald ${ }^{12}$, C. McCoey ${ }^{17}$, I. Mehdi ${ }^{12}$, A. Megej ${ }^{21}$, M. Melchior ${ }^{11}$, L. Meinsma ${ }^{1}, \mathrm{H}_{\text {. Merkel }}{ }^{10}$, M. Michalska ${ }^{23}$, C. Monstein ${ }^{11}$, D. Moratschke ${ }^{5}$, P. Morris ${ }^{35}$,

H. Muller ${ }^{5}$, J. A. Murphy ${ }^{26}$, A. Naber ${ }^{1}$, E. Natale ${ }^{32}$, W. Nowosielski ${ }^{23}$, F. Nuzzolo ${ }^{16}$, M. Olberg ${ }^{1,10}$, M. Olbrich ${ }^{5}$, R. Orfei ${ }^{16}$, P. Orleanski ${ }^{23}$, V. Ossenkopf ${ }^{1,5}$, T. Peacock ${ }^{26}$, J. C. Pearson ${ }^{12}$, I. Peron ${ }^{19,20,31}$, S. Phillip-May ${ }^{15}$, L. Piazzo ${ }^{16}$, P. Planesas ${ }^{3,9}$, M. Rataj ${ }^{23}$, L. Ravera ${ }^{6,7}$, C. Risacher ${ }^{18}$, M. Salez ${ }^{19,20}$, L. A. Samoska ${ }^{12}$, P. Saraceno ${ }^{16}$, R. Schieder ${ }^{5}$,

E. Schlecht ${ }^{12}$, F. Schlöder ${ }^{5}$, F. Schmülling ${ }^{5}$, M. Schultz ${ }^{5}$, K. Schuster ${ }^{31}$, O. Siebertz ${ }^{5}$, H. Smit $^{1}$, R. Szczerba ${ }^{25}$,

R. Shipman ${ }^{1}$, E. Steinmetz ${ }^{18}$, J. A. Stern ${ }^{12}$, M. Stokroos ${ }^{1}$, R. Teipen ${ }^{5}$, D. Teyssier ${ }^{1,33}$, T. Tils ${ }^{5}$, N. Trappe ${ }^{26}$, C. van Baaren ${ }^{1}$, B.-J. van Leeuwen ${ }^{1}$, H. van de $\operatorname{Stadt}^{1}$, H. Visser ${ }^{27}$, K. J. Wildeman ${ }^{1}$, C. K. Wafelbakker ${ }^{1}$, J. S. Ward ${ }^{12}$,

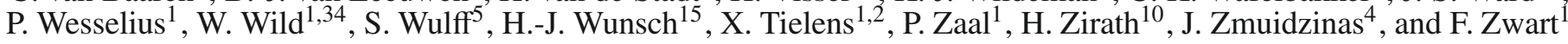

(Affiliations are available in the online edition)

Received 1 April 2010 / Accepted 13 May 2010

\section{ABSTRACT}

Aims. This paper describes the Heterodyne Instrument for the Far-Infrared (HIFI) that was launched onboard ESA's Herschel Space Observatory in May 2009.

Methods. The instrument is a set of 7 heterodyne receivers that are electronically tuneable, covering 480-1250 GHz with SIS mixers and the 1410-1910 GHz range with hot electron bolometer (HEB) mixers. The local oscillator (LO) subsystem comprises a Ka-band synthesizer followed by 14 chains of frequency multipliers and 2 chains for each frequency band. A pair of auto-correlators and a pair of acousto-optical spectrometers process the two IF signals from the dual-polarization, single-pixel front-ends to provide instantaneous frequency coverage of $2 \times 4 \mathrm{GHz}$, with a set of resolutions $(125 \mathrm{kHz}$ to $1 \mathrm{MHz})$ that are better than $0.1 \mathrm{~km} \mathrm{~s}^{-1}$.

Results. After a successful qualification and a pre-launch TB/TV test program, the flight instrument is now in-orbit and completed successfully the commissioning and performance verification phase. The in-orbit performance of the receivers matches the pre-launch sensitivities. We also report on the in-orbit performance of the receivers and some first results of HIFI's operations.

Key words. instrumentation: spectrographs - methods: observational - infrared: general - submillimeter: general - techniques: spectroscopic

\section{Introduction}

HIFI, the Heterodyne Instrument for the Far-Infrared, is one of the three instruments onboard the Herschel Space Observatory (Pilbratt et al. 2010). Herschel was launched in May 2009, and because it is an observatory-type mission where HIFI

* Herschel is an ESA space observatory with science instruments provided by European-led Principal Investigator consortia and with important participation from NASA. provides its high spectral resolution capability, HIFI needs to be versatile enough to be able to address many key themes in modern astrophysics. The instrument is therefore designed to provide very high spectral resolution over the widest possible frequency range. With the limited collecting area of a $3.5 \mathrm{~m}$ telescope, state-of-the-art superconducting mixers with near quantum-noise limit system noise temperatures are required for sensitivity. With the very high spectral resolution provided by the heterodyne radio technique, HIFI will spectrally 


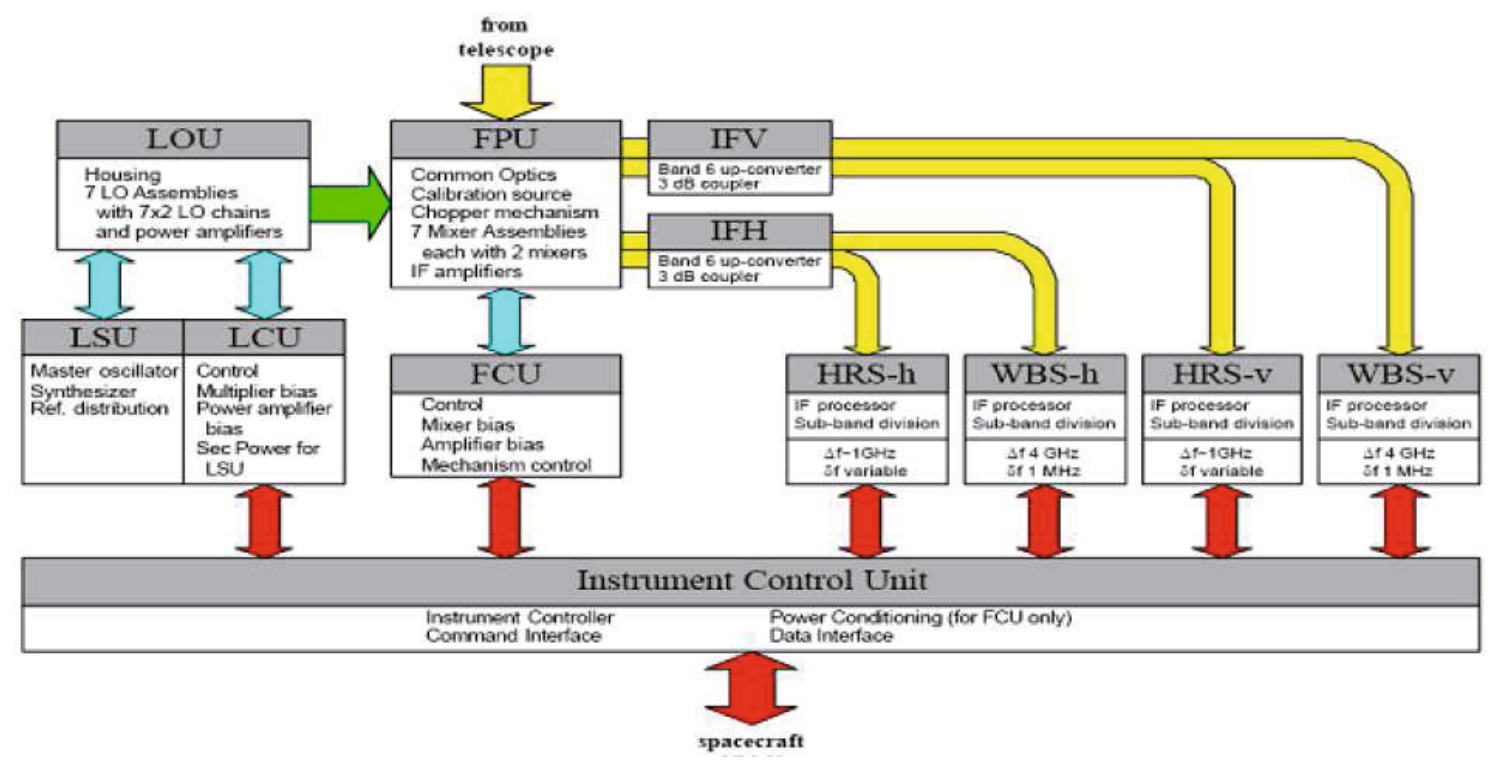

Fig. 1. HIFI block-diagram showing the various subsystems and their interconnections.

disentangle the contribution from the various emission regions covered with the relatively large beam of the Herschel telescope. In this paper we describe the key design features, the observing modes, the in-orbit receiver sensitivity, and some first spectra taken. Details of the calibration and flight performance are described by Roelfsema et al. (in prep.).

\section{Scientific rationale for the HIFI design}

The scientific themes for HIFI are mainly related to the understanding of the cyclic interrelation of stars and the interstellar medium in galaxies. On the one hand, stars - and planetary systems - are formed through gravitational collapse of interstellar molecular clouds. On the other hand, the interstellar medium is composed from the ejecta - enriched by newly synthesized elements - of dying stars. This complex interplay between stars and the ISM drives the evolution, hence the observational characteristics, of the Milky Way and other galaxies, all the way back to the earliest protogalaxies at high redshift. Although HIFI will have capabilities that address many key topics in modern astrophysics, there are three areas for which HIFIs design was optimized and will be unique: 1) observations of the water lines lines ending in the ground states that are essential for absorption studies of cold water, falling in bands $1(557 \mathrm{GHz})$, $4(1.11 \mathrm{THz})$, and $6(1.67 \mathrm{THz}) ; 2)$ a survey of the molecular complexity of the Universe; and 3) observations of (redshifted) ionized Carbon [CII] at $1.9 \mathrm{THz}$ (band 7). These three topics required the high spectral resolution and the wide frequency range given below.

\section{HIFI instrument design concept}

By combining the radio heterodyne technique with near quantum-noise limited sensitivity from superconducting detector technology, applying state-of-the-art in microwave technology, and the latest development in correlator and AOS spectrometer technology, it was possible to construct an instrument with the following capabilities: a) continuous frequency coverage from 480 to $1250 \mathrm{GHz}$ in five bands, while 2 bands will provide coverage for 1410-1910 GHz; b) spectral resolutions between 300 and $0.03 \mathrm{~km} \mathrm{~s}^{-1}$; and c) detection sensitivity close to the fundamental quantum noise limit. HIFI instrument consists of five major subsystems, shown in the blockdiagram of Fig. 1.

1. The focal-plane subsystem comprises the focal-plane unit (FPU) mounted at the optical bench on top of the liquid $\mathrm{He}$ vessel inside the cryostat. An FPU control unit (FCU), located at the service module (SVM), supplies the bias voltages for mixers and IF preamplifiers in the FPU, and controls the LO diplexers, the focal plane chopper mechanism, and the calibration source.

2. The LO sub-system comprises the local oscillator unit (LOU), located on the outside wall of the Herschel cryostat. The LOU contains 7 local oscillator assemblies (LOA), each containing two LO multiplier chains. These chains are fed by a common LO source unit (LSU) followed by triplers, power amplifiers, and multipliers that generate the LO signals that are coupled into the FPU via 7 windows in the cryostat wall. The LSU and local oscillator control unit (LCU) are located in the SVM and contain the reference frequency source, the bias supplies, and controls of the local oscillator.

3. The wide-band spectrometer (WBS) consists of a pair of array acousto-optical spectrometers (AOS) with a frequency resolution of about $1 \mathrm{MHz}$ and a bandwidth of $4 \mathrm{GHz}$ for each of the two polarizations.

4. The high-resolution spectrometer (HRS) is a pair of autocorrelator spectrometers, divided into sub-bands with several combinations of bandwidth and frequency resolutions (see Fig. 6). Each sub-band can be placed anywhere within the full $4 \mathrm{GHz}$ IF band.

5. An instrument control unit (ICU) within the SVM interprets commands from the satellite tele-command system, controls the operation of the instrument, and returns science and housekeeping data to the satellite telemetry system.

\section{Focal plane subsystem}

The focal-plane unit (FPU) (Jackson et al. 2002) is the part of the focal plane subsystem located inside the Herschel cryostat. The FPU produces seven optical beams (one per band) that are spatially separated in the focal plane, resulting in an angular separation of the beams on the sky. The FPU contains the common 


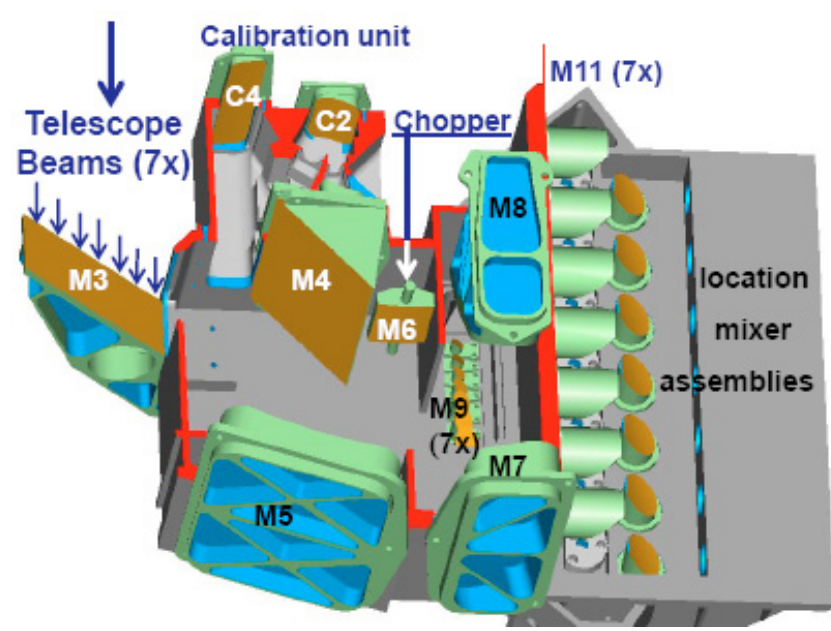

Fig. 2. Common optics assembly layout. Mirrors M3, 4, 5 provide an image of the telescope secondary at the chopper mirror M6. Mirrors M7, 8 , and 9 provide the channel splitting optics to increase the beam separation between the mixer bands.

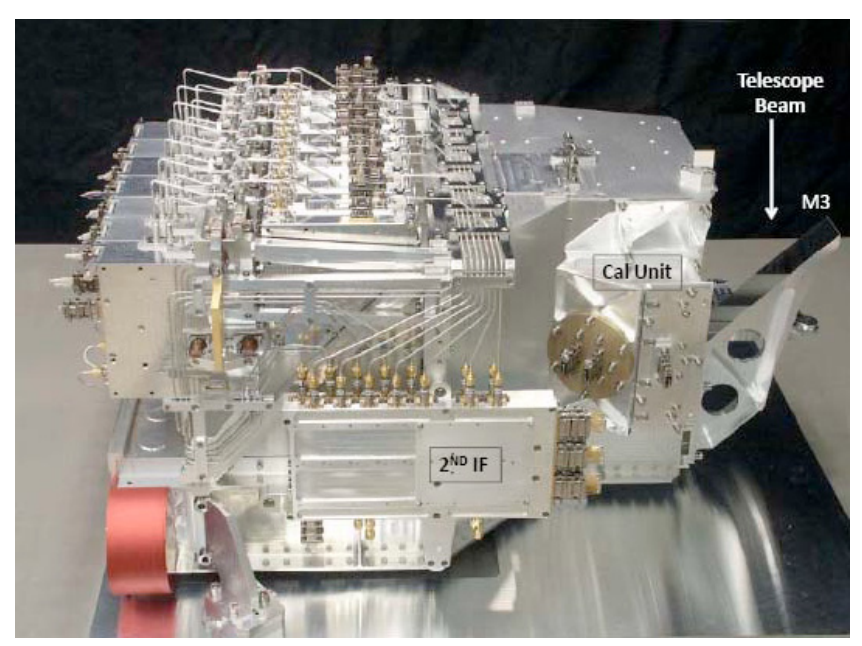

Fig. 3. The flight model focal plane unit showing the input mirror (M3) and the 2nd IF amplifier box and Calibration module.

optics assembly (COA), which is the basis of the FPU structure (see Fig. 2). It consists of three optical blocks: the telescope relay optics, the band splitting optics, and the cold LO optics. The relay optics for the sky signals that is used for all mixer bands includes a focal plane chopper and optics for the calibration sources. The FPU houses the 7 pairs of mixer subassemblies (MSA) with their specific input optics and a common low-noise IF amplifier box with 2 sets of seven amplifiers. In each set of amplifiers the seven outputs are combined with microwave couplers into one output. Since only one mixer band is operating at a time there is no need for activated switches to decouple the other 6 , thus avoiding unwanted failure modes. The 7 LO beams enter from the back of the FPU via the cold LO optics, producing frequency independent waists in the diplexer boxes positions (located at the cryostat window) and where alignment can be carried out with visible light since the optics is all reflective.

The same aluminum material is used for all the mirrors, as well as for the mechanical structure. Figure 3 shows a picture of the flight model of the FPU after the final assembly.

There are 7 mixer bands (see Fig. 4) that cover the overall HIFI frequency range, each with two mixer sub-assemblies (MSA) and a diplexer assembly. One mixer band is operational

\begin{tabular}{|c|c|c|c|c|}
\hline $\begin{array}{l}\text { Mixer Band/ } \\
\text { Laboratories }\end{array}$ & $\begin{array}{l}\text { Frequency } \\
\text { range: GHz }\end{array}$ & Mixer element & $\begin{array}{l}\text { Matching } \\
\text { circuit }\end{array}$ & $\begin{array}{l}\text { Feed and coupling } \\
\text { structure }\end{array}$ \\
\hline $\begin{array}{c}1 \\
\text { LERMA/IRAM }\end{array}$ & $480-640$ & $\begin{array}{c}\text { SIS } \\
\mathrm{Nb}-\mathrm{Al}_{2} \mathrm{O}_{3}-\mathrm{Nb}\end{array}$ & $\begin{array}{l}\mathrm{Nb} \text { on } \mathrm{Nb} \\
\text { microstrip }\end{array}$ & $\begin{array}{l}\text { corrugated horn } \\
\text { and waveguide }\end{array}$ \\
\hline $\begin{array}{c}2 \\
\text { KOSMA }\end{array}$ & $640-800$ & $\begin{array}{c}\text { SIS } \\
\text { Nb- } \mathrm{Al}_{2} \mathrm{O}_{3}-\mathrm{Nb} \\
\end{array}$ & $\begin{array}{l}\mathrm{Nb} \text { on } \mathrm{NbTiN} \\
\text { microstrip }\end{array}$ & $\begin{array}{l}\text { corrugated horn } \\
\text { and waveguide }\end{array}$ \\
\hline $\begin{array}{c}3 \\
\text { SRON/TUD }\end{array}$ & $800-960$ & $\begin{array}{c}\text { SIS } \\
\text { Nb-Al } \mathrm{O}_{2}-\mathrm{Nb} \\
\end{array}$ & $\begin{array}{c}\text { Al on NbTiN } \\
\text { microstrip }\end{array}$ & $\begin{array}{l}\text { corrugated horn } \\
\text { and waveguide }\end{array}$ \\
\hline $\begin{array}{c}4 \\
\text { SRON/TUD } \\
\end{array}$ & $960-1120$ & $\begin{array}{c}\text { SIS } \\
\mathrm{Nb}-\mathrm{Al}_{2} \mathrm{O}_{3}-\mathrm{Nb} \\
\end{array}$ & $\begin{array}{c}\text { Al on NbTiN } \\
\text { microstrip }\end{array}$ & $\begin{array}{c}\text { corrugated horn } \\
\text { and waveguide }\end{array}$ \\
\hline $\begin{array}{c}5 \\
\text { CalTech/JPL }\end{array}$ & $1120-1250$ & $\begin{array}{c}\text { SIS } \\
\text { Nb-AlN-NbTiN }\end{array}$ & $\begin{array}{l}\text { Au on Nb } \\
\text { microstrip }\end{array}$ & $\begin{array}{c}\text { lens and twin slot } \\
\text { planar antenna }\end{array}$ \\
\hline $\begin{array}{c}6 \\
\text { CTH/JPL }\end{array}$ & $1410-1703$ & $\begin{array}{c}\text { HEB NbN } \\
\text { phonon cooled }\end{array}$ & & $\begin{array}{c}\text { lens and twin slot } \\
\text { planar antenna }\end{array}$ \\
\hline $\begin{array}{c}7 \\
\mathrm{CTH} / \mathrm{JPL}\end{array}$ & $1703-1910$ & $\begin{array}{c}\text { HEB NbN } \\
\text { phonon cooled }\end{array}$ & & $\begin{array}{l}\text { lens and twin slot } \\
\text { planar antenna }\end{array}$ \\
\hline
\end{tabular}

Fig. 4. Overview of mixer materials and implemented antenna technology. Both types of mixers, SIS and HEB, are operating at $1.7 \mathrm{~K}$.

at a time, with two mixers operating at orthogonal polarizations. This also provides redundancy for the frequency bands. The MSAs contain mechanical supports, mixers, diplexers, and polarizers, as well as IF pre-amplifiers, and they are mechanically mounted on the FPU.

To have the HIFI mixers cover such a wide frequency range, several dedicated developments were needed to optimize the sensitivity for each frequency band. The mixers have been developed in several European and US laboratories, each optimizing the technology required for its band. For a summary, see Fig. 4 where the details of the mixer elements are given with the materials of the matching circuits and the feed and coupling structures (Cherednichenko et al. 2005; Delorme et al. 2005; Jackson et al. 2006; Teipen et al. 2004).

An FPU control unit (FCU), located at the SVM, supplies the bias voltages for mixers and IF preamplifiers, and drives and controls a) the diplexers for LO injection; b) the focal plane chopper mechanism and c) the calibration source.

\section{The local oscillator subsystem}

The LO multiplication scheme is illustrated in Fig. 5 (Pearson et al. 2000). Each mixer frequency band is covered by two chains in the corresponding LOAs. The tuning ranges are achieved with a broadband, high-power mm-wave amplifier as input source for the varactor frequency multiplier chains. The demonstrated output powers of the amplifiers are sometimes over $400 \mathrm{~mW}$ in the 75-100 GHz frequency range. Planar Schottky diodes are used for all the stages of the multiplier chains (Ward et al. 2003). These not only provide high power-handling capability and a wide bandwidth, but improved also considerably the reproducibility and stability, needed for a satellite project.

\section{The HIFI spectrometers}

For the HIFI spectrometers, two techniques were applied in order to cover the resolution and frequency coverage requirements. The digital autocorrelator (HRS) provides a very high spectral resolution over limited bandwidth and has a high flexibility, while the acousto-optical technique, used in the WBS, provides a wide frequency coverage for low power and low mass. 


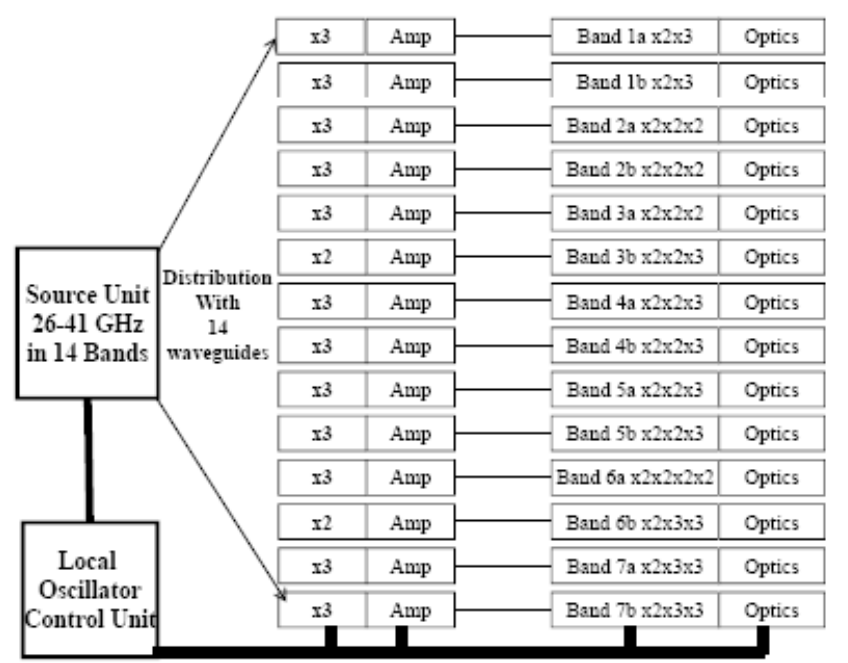

Fig. 5. The LO subsystem block diagram including the multiplication scheme as used in HIFI.

\begin{tabular}{|c|c|c|c|c|c|}
\hline Mode & $\begin{array}{c}\text { HRS - High } \\
\text { Resolution }\end{array}$ & $\begin{array}{c}\text { HRS - Normal } \\
\text { Resolution }\end{array}$ & $\begin{array}{c}\text { HRS - Low } \\
\text { Resolution }\end{array}$ & $\begin{array}{c}\text { HRS - Wide } \\
\text { Band }\end{array}$ & WBS \\
\hline $\begin{array}{c}\text { Number of } \\
\text { Bands }\end{array}$ & 1 & 2 & 4 & 8 & 1 \\
\hline $\begin{array}{c}\text { Bandwidth } \\
\text { (MHz) }\end{array}$ & 235 & 235 & 235 & 235 & 4000 \\
\hline $\begin{array}{c}\text { FWHM } \\
(\text { MHz) }\end{array}$ & 0.125 & 0.25 & 0.5 & 1.0 & 1.1 \\
\hline
\end{tabular}

Fig. 6. Overview of the spectral resolution and frequency coverage of the HIFI spectrometers HRS and WBS and the number of frequency bands.

\subsection{The high resolution spectrometer (HRS)}

The HRS of HIFI is a set of digital autocorrelation spectrometers (Belgacem et al. 2003). The IF input signal is analyzed in sub-bands of $235 \mathrm{MHz}$ width, after an analog down-conversion. Their bandwidth and resolution, as realized in the HRS flight units, are summarized in Fig. 6. The strategy for the HRS is to have real-time signal processing functions at electronic level onboard and the software signal processing on the ground. After analogue processing and digitization, the autocorrelation functions of the input signal are computed with correlation modules made up of application specific ICs (ASIC). These correlation functions are sent via the ICU to the ground to be processed.

There are three software modules for the HRS: 1) specific processing of the autocorrelation spectrometers to obtain the power spectrum from the input signal autocorrelation function; 2) power calibration processing to calibrate the observed spectrum in terms of power; 3 ) a set of routines to test and characterize the HRS.

\subsection{The HIFI wideband spectrometer (WBS)}

The WBS uses the acousto-optical principle of light diffraction on a phonon wave created by the IF signal in a Bragg crystal to realize a compact, low-power, and wide bandwidth spectrometer. A detailed description of an Array AOS as used in HIFI is given by Horn et al. (1999). The HIFI WBS is described specifically by Schieder et al. (2002). The WBS optical layout is shown in Fig. 7, together with a picture of the optical unit of the flight model. Because the maximum bandwidth of acousto-optical deflectors is relatively limited, four parallel $1 \mathrm{GHz}$ units, integrated into a single optics setup, are used to obtain the full frequency coverage of $4 \mathrm{GHz}$. For this, an IF processor $(4 \mathrm{GHz}$ to $1 \mathrm{GHz}$ )

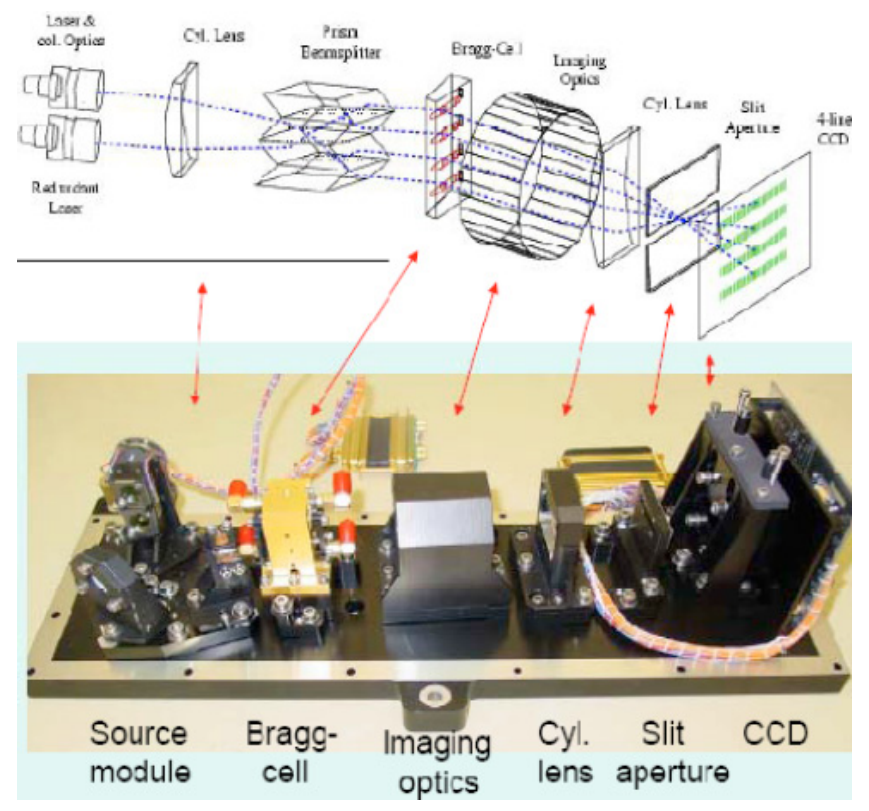

Fig. 7. The WBS optical flight module with the optical layout.

was designed followed by a novel Bragg cell with four transducers, each covering $1 \mathrm{GHz}$. The CCD layout was adapted to the 4 transducer layout and the compactness of the unit minimizes thermal effects on the spectral baseline. The resulting spectral resolution for the HIFI WBS is $1.1 \mathrm{MHz}$.

\section{The HIFI instrument control unit}

The ICU is the only subsystem that interfaces electrically with the spacecraft for telemetry and commanding. It also distributes electrical power to the FCU, takes care of the command execution and synchronization, packages the telemetry $\left(\sim 100 \mathrm{kbit} \mathrm{s}^{-1}\right)$, and provides the health-autonomous mode. The ICU is positioned in the warm part of the $\mathrm{S} / \mathrm{C}$, close to the FCU, LCU, WBS-V, WBS-H, HRS-V, and HRS-H subsystems.

\section{Observing modes and AOTs}

\subsection{HIFI observing modes}

The implemented observing modes for the Herschel-HIFI instrument have been constructed around possible ways to take near-real-time reference spectra to correct for the minimized, but unavoidable, drifts in the relative spectral response of the IF pass-band of the heterodyne spectrometer. These reference modes are a) position switch; b) dual beam switch; c) frequency switch; and d) load chop. These reference modes are used for the three HIFI astronomical observing templates (AOTs): 1) singlepoint observations; 2) mapping observations; and 3) spectral scans. A summary of the combinations of observing modes and AOTs is given in Fig. 8. Details are in Roelfsema et al. (in prep.).

\subsubsection{Position switch and dual beam switch (DBS)}

In position switch mode, the HIFI beam is pointed alternately at a target position and at a reference position. The reference position is usually chosen to be a nearby area of the sky that is devoid of emission in the band being used. If the reference position also has emission, then the reference position must be calibrated too. In $D S B$, an internal chopper mirror within HIFI (M6 in Fig. 2) 


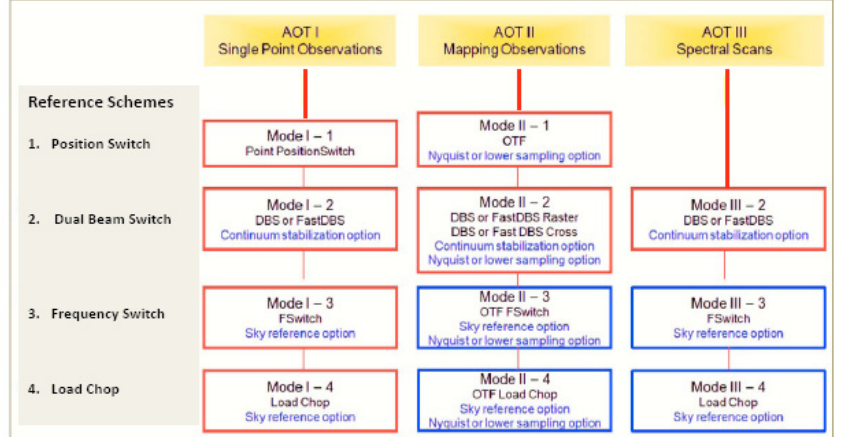

Fig. 8. Overview of HIFI observing modes and AOTs as they are implemented in the satellite commanding and data reduction software.

is used to move the beam to a reference OFF position on the sky within 3 arcmin away from the on-target position. Since moving the internal mirror changes the light path for the incoming waves, the possibility of residual standing waves exists. By moving the telescope so that the source appears alternatively in both $\mathrm{ON}$ and OFF chop positions, the impact of standing wave differences is eliminated to a first order, see Roelfsema et al. (in prep.). Two chopper speeds are implemented.

\subsubsection{Frequency switch}

In this mode, following an observation at a given on frequency, the local oscillator frequency is changed by a small amount (a few tens of MHz). The shift in frequency is small enough that the lines of interest remain observable at the two LO frequencies. This therefore makes for a very efficient mode since target emission lines are observed in both ON and OFF positions. Adapting the frequency throw to the period of the dominant standing wave leads to substantial reduction of the baseline ripple.

\subsubsection{Load chop}

In this reference scheme, the HIFI internal cold source is used as a reference. The chopping mirror alternately looks at the target on the sky and the internal load. This is particularly useful when there are no emission-free regions near the target position or in the frequency domain.

\subsection{HIFI astronomical observing templates (AOT)}

\subsubsection{Single-point AOT}

This AOT is designed for pointed observations. It can utilize all four observing modes as described above. In the case of frequency switch and load chop, an option exists that allows to additionally observe an OFF position away from the on-target line-of-sight.

\subsubsection{Mapping AOT: on-the-fly (OTF) and raster mapping}

OTF mapping is probably the most efficient means of collecting data to map emission over a large region of sky. Data are taken continuously while the telescope is scanned back and forth across the target, with data readouts taking place at a scanning distance similar to the beam size at the frequency of observation. A single emission-free point reference position measurement is used as an off measurement. Frequency switching and load chop are also available in combination with OTF mapping.

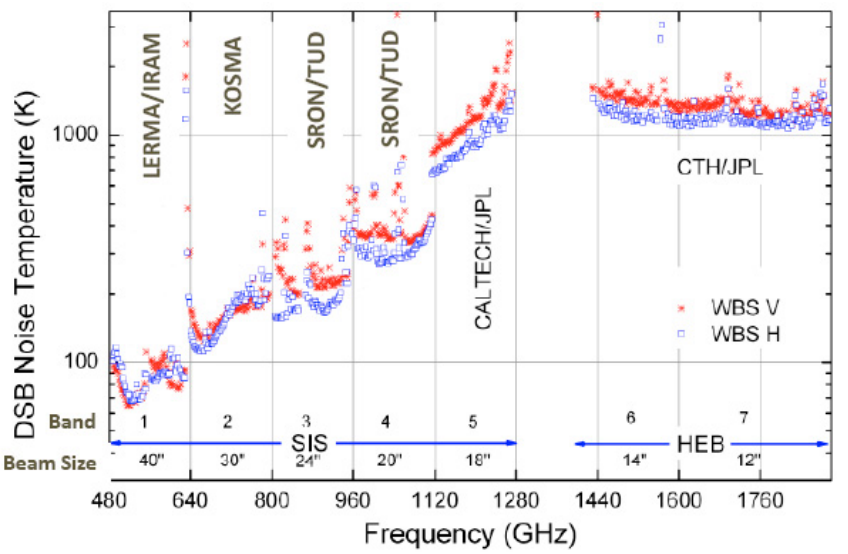

Fig. 9. HIFI's $T_{\text {sys }}$ DSB for $H$ and $V$ polarization for the 7 bands, together with their aimed angular resolution (HPBW). Also shown are the institutes that provided the mixers.

When raster mapping is carried out, the DBS is the only available mode.

\subsubsection{Spectral scans AOT}

This AOT is designed for making spectral scans for a part or the whole of a frequency band. Typically, these are made at LO frequencies that are about $1 \mathrm{GHz}$ apart. There are three reference schemes available here: DBS, frequency switch, and load chop. As for the single point AOT, the frequency switch and load chop case offer the option additionally observing an OFF position. For each observing frequency setting, measurements can be made, resulting in fully calibrated, dual sideband spectra at each of the LO settings. The creation of a single sideband spectrum is afterwards achieved by a deconvolution routine during data processing.

\section{HIFI In-orbit performance}

\subsection{Tests and observations}

The functional tests and calibrations have indicated that the scientific capabilities are close to what was expected or even exceed the prelaunch results. Figure 9 shows the in-orbit system noise temperatures for the 5 SIS and the two HEB bands for both linear polarizations. The data were taken with the WBS spectrometers.

A detailed report of the in-orbit performance will be given by Roelfsema et al. (in prep.) in the dedicated A\&A HIFI First Results issue. A HIFI spectral scan of band $4 \mathrm{~b}$ from $1066-1107 \mathrm{GHz}$ towards the Orion hot core $\left(\alpha_{\mathrm{J} 2000}=\right.$ $5^{\mathrm{h}} 35^{\mathrm{m}} 14.3^{\mathrm{m}}$ and $\left.\delta_{J 2000}=-5^{\circ} 22^{\prime} 36.7^{\prime \prime}\right)$ is presented in Fig. 10. These data were obtained during HIFI performance verification observations as part of the guaranteed time key program Herschel/HIFI Observations of Extraordinary Sources: The Orion and Sagittarius B2 Starforming Regions (HEXOS). The observations utilize the DBS mode and the WBS spectrometer with a spectral resolution of $1.1 \mathrm{MHz}$. The band $4 \mathrm{~b}$ spectral scan consists of double sideband spectra with a redundancy of 12 , which provides observations of a given lower or upper sideband frequency with 12 different settings of the local oscillator. This allows for the deconvolution and isolation of single sideband spectra. In this instance, we applied the standard HIFI deconvolution using the doDeconvolution task within HIPE. 


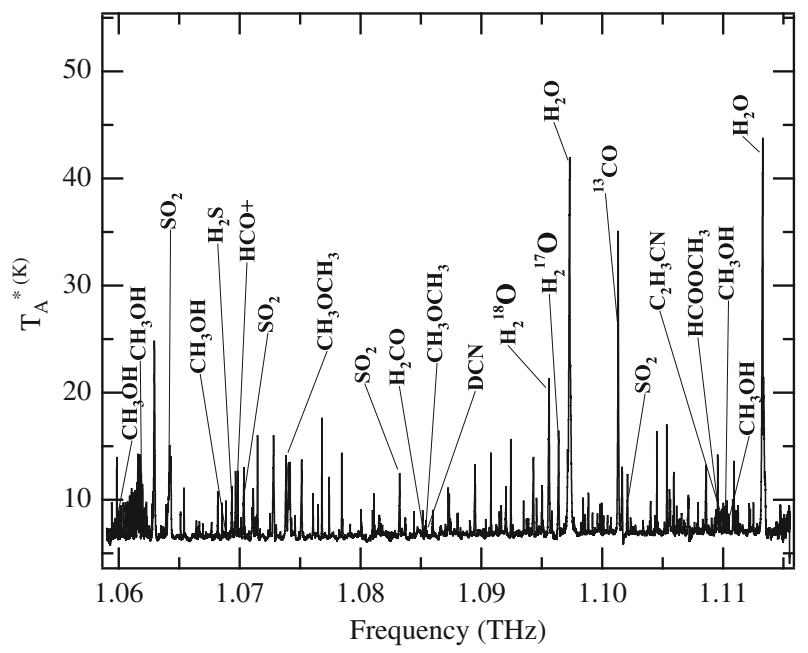

Fig. 10. Deconvolved HIFI spectral scan of band $4 \mathrm{~b}$ from 1066-1107 GHz towards the Orion hot core (courtesy HEXOS team and ESA).

\subsection{Instrument status}

On observational day 81 (August 2, 2009), telemetry from Herschel showed that HIFI had entered an anomalous state. The housekeeping from the LCU contained no information anymore, the power consumption of the LCU had suddenly dropped, and the temperature of the LCU panel started decreasing. A thorough investigation of the problem started. After evaluating and eliminating many scenarios for the anomaly, the only possible series of events emerged: a single event upset, caused by a cosmic particle hit, in the LCU memory led the LCU microcontroller to jump from address to address, finally ending at address zero, which implied pulling the standby-switch. Pulling this switch on a fully-powered instrument led to a voltage transient in the LCU, which made one of the secondary rectifier diodes in one of the DCDC converters fail. A switch to the redundant LCU (and ICU, FCU) was needed. Together with adaptations of the software to prevent pulling the standby-switch, HIFI is now operating safely again.

Acknowledgements. The Herschel-HIFI instrument has been constructed, tested and prepared for operations by a large set of teams of dedicated engineers, scientists, and managers from 12 European and North American countries, with additional funding by grants from their national space agencies and science foundations. We acknowledge the support for the Netherlands by NWO; for Germany by grants $500 F 000-1 / 2 / 5$ of the Deutsches Zentrum für Luft- und Raumfahrt (DLR) and by central resources of the Max-Planck-Society. For the USA a portion of this research was performed at the JPL, California Institute of Technology, under contract with the National Aeronautics and Space Administration and part of the NASA Herschel Science Center guaranteed-time observer program was provided by NASA; for France by the Centre National de la Recherche Scientifique/Institut National des Sciences de l'Univers (CNRS/INSU) and Centre National d'Etudes Spatiales (CNES); for Italy by Agenzia Spaziale Italiana - ASI, Contract I/005/07/0, and the Istituto Nazionale di Astrofisica INAF; for Canada by the Canadian Space Agency. For Poland this work has been partly supported by grant N203 393334 of the Science and High Education Ministry of Poland; for Spain support came from Ministerio de Ciencia e Innovacin (Consejo de Investigaciones Cientificas) and Ministerio de Fomento (Instituto Geográfico Nacional); for Sweden support came from the Swedish National Space Board under grant numbers Dnr 136/04, Dnr 114/07;
Dnr 286/05; Dnr 116/03, and by Knut and Alice Wallenberg Foundation. For Ireland support was provided by Enterprise Ireland through a number of Prodex grants. The home institutes have invested many resources into this project. Their contributions and continuous support are deeply appreciated by the HIFI consortium. Also the fruitful collaborations with ESA and the Herschel and HIFI industrial partners are acknowledged. This extremely challenging project is a team effort based upon the creativity, dedication, and perseverance of many individuals participating in this scientific and technological enterprise, amongst others: W. Aalders, A. Abrams, W. Ader, C. Amoros, G. Arbery, O. Armengaud, J. Baker, P. Baldetti, P. Barras, T. Bartels, A. Baryshev, A. Baudry, G. Beaudin, A. Beckers, M. Belgacem, C. Berthod, N. Biver, Y. Blanc, J. Bleeker, M. Bonenkamp, A. Boner, F. Boulanger, J.-C. Bouquier, H. Braafhart, J. Braine, M. Bruijn, C. Bruineman, P. Bruneau, N. Bruning, A. Campbell, M. Carter, C. Casteels, C. Chappert, P. Chavatte, W. Chun, J. Couterets, P. Crosby, J.-P. Crussaire, A. Csillaghy, Y. Cupissol, E. Dartois, F. Dauplay, N. Giurleo, R. de Haan, Doug Johnstone, T. de Jong, A. de Kleine, R. de la Rie, R. de Lange, J.-H. de Raignac, D. Deboffle, L. deJong, K. Deng, J. Dercksen, A. Deschamps, J. Diez Gamero, H. Doedens, C. du Maine, P. Encrenaz, R. Ferber, A. Feret, F. Flederus, M. Frerking, A. Fung, S. Gadomski, R. Garcia-Nogal, R. Gathier, T. Harper, L. Hotte, J. Gavira Izquierdo, N. Gehniau, A. Girard, F. Glize, J.-M. Glorian, G. Grund, S. Halleguen, D. Harding, F. Herpin, G. Hiemstra, L. Hiemstra, N. Hoac, W. Horinga, M. Houde, W. Janssen, H. Janzen, M. Jochemsen, G. Juchnikowski, A. Karl, S. Kikken, T. Kirst, P. Kohsiek, J. Koops van het Jagt, M. Kroug, B. Kuip, D. Lagrange, J. Lankwaarden, J.-M. Larr, P. Laubert, A. Lecacheux, B. Lecomte, E. Lecomte, K. Liao, Y. Longval, A. Loung, P. Lowes, Peter Martin, G. Martinez-Medina, H. E. Matthews, S. Matthias, J.-Y. Mayvial, W. McCutcheon, W. McGrath, K. Mercier, D. Miller, D. Monnier, G. Montignac, A. Morbidini, R. Moreno, P. Mueller, J. Newell, T. Newman, A. Nieuwenhuizen, J. Novag, H. Ode, A. Orzati, J. Panman, M. Paquette, G. Parks, A. Peralta, M. Perault, A. Perez Lopez, S. Phillips, E. Pizzi, G. Ploeger, Rene Plume, D. Pukala, J.-R. Rabasse, P. Ramon, M. Rataj, J. Recine, M. Ridder, R. Roelfsema, D. Romefort, C. Rosolen, L. Roucayrol, M. Rudin, K. Saad, W. Salomons, E. Sanchez, M. Schekkerman, R. Schuurhof, N. Snijders, J. Spatazz, J. Swift, B. Thomas, A. Tizon, M. Torres, C. Troung, R. Tsang, J. van der Eb, A. van der Horst, K. van der Hucht, R. van der Schuur, P. van Leeuwen, W. van Leeuwen, D. Van Nguyen, J. van Veldhuizen, H. van Weers, C. Vastel, J. Veenendaal, J. Velebir, H. Wang, D. Warden, S. Weinreb, M. Wells, K. Wielinga, M. Winkler, G. Winnewisser, S. Withington, Q. Xie, P. Yocom and A. Zijlstra. Thanks to their efforts the instrument could be tested and delivered to ESA and its scientific community. We are gratefull to the astronomers of the HIFI Key-Program teams and their Principal Investigators, in particular E. van Dishoeck, E. Bergin, C. Ceccarelli, M. Gerin, V. Bujarrabal, whose imagination about what HIFI would be able to observe helped us to get through the difficult phases in the HIFI development program. Also many thanks go to the members of the various FIRST/Herschel Science Teams and ESA Project Teams that worked for this mission and in particular to G. Winnewisser, H. Olthof, R. Genzel, U. Frisk, S. Volonte, G. Pilbratt, J. Riedinger, Th. Passvogel, C. Schamberg, and G. Crone.

\section{References}

Belgacem, M., Ravera, L., Caux, E., Caïs, P., \& Cros, A. 2003, New Astron., 9, 43

Cherednichenko, S., Drakinskiy, V., \& Berg, T. 2005, Proc. Europ. Microwave Assoc., 1, 3

Delorme, Y., Salez, M., Lecomt, B., et al. 2005, Proc. 16th ISSTT (Göteborg, Sweden), 444

Horn, J., Siebertz, O., Schmülling, F., et al. 1999, Exper. Astron., 9, 17

Jackson, B. D., Wildeman, K. J., \& Whyborn, N. D. 2002, on behalf of the HIFI

Focal Plane Consortium, Proc. 13th ISSTT, Cambridge, MA, USA, 339

Jackson, B. D., de Lange, G., Zijlstra, T., et al. 2006, IEEE Trans. MTT, 54, 547 Pearson, J. C., Güsten, R., Klein, Th., \& Whyborn, N. D. 2000, SPIE, 4013, 264 Pilbratt, G. L., et al. 2010, A\&A, 518, L1

Schieder, R., Siebertz, O., Gal, C., et al. 2002, SPIE, 4855, 313

Teipen, R., Justen, M., Tils, T., et al. 2005, Proc. 16th ISSTT, Göteborg, Sweden, 199

Ward, J. S., Chattopadhyay, G., Maestrini, A., et al. 2003, Proc. 14th ISSTT, Charlottesville, USA, 94 
1 SRON Netherlands Institute for Space Research, PO Box 800, Groningen, The Netherlands

2 Leiden Observatory, University of Leiden, The Netherlands

3 Joint Alma Observatory, Santiago, Chile e-mail: tdegraau@alma.cl

${ }^{4}$ Physics Department, California Institute of Technology, Pasadena, CA, USA

5 KOSMA, I. Physik. Institut, Universität zu K'öln, Zülpicher Str. 77, 50937 Köln, Germany

${ }^{6}$ Centre d'Etude Spatiale des Rayonnements, Université de Toulouse [UPS], 31062 Toulouse Cedex 9, France

7 CNRS/INSU, UMR 5187, 9 Avenue du Colonel Roche, 31028 Toulouse Cedex 4, France

8 Observatorio Astronómico Nacional (IGN), Calle Alfonso XII 3, 28014 Madrid, Spain

9 Observatorio Astronómico Nacional (IGN), Centro Astronómico de Yebes, Apartado 148. 19080 Guadalajara, Spain

${ }_{10}$ Chalmers University of Technology, 41296 Göteborg, Sweden

11 Astronomical Institute, ETH, Zürich, 8093 Zürich, Switzerland

12 Jet Propulsion Laboratory, 4800 Oak Grove Drive, MC 168-314, Pasadena, CA 91109 USA

13 Université de Bordeaux, Laboratoire d'Astrophysique de Bordeaux, 33000 Bordeaux, France

14 CNRS/INSU, UMR 5804, BP 89, 33271 Floirac, France

15 MPI für Radio Astronomie, Auf dem Hügel 69, 53121 Bonn, Germany

16 Istituto Fisica Spazio Interplanetario INAF, via Fosso del Cavaliere 100, 00133 Roma, Italy

17 Department of Physics and Astronomy, University of Waterloo, Waterloo, ON N2L 3G1, Canada

18 MPI für Sonnensystemforschung, 37191 Katlenburg-Lindau, Germany

19 Laboratoire d'Etudes du Rayonnement et de la Matière en Astrophysique, UMR 8112 CNRS/INSU, OP, ENS, UPMC, UCP, Paris, France
${ }^{20}$ LERMA, Observatoire de Paris, 61 Avenue de l'Observatoire, 75014 Paris, France

21 Institute für Hochfrequenz Techniques, ETH, Zürich, 8093 Zürich, ETH HF, Switzerland

22 Department of Astronomy, Stockholm University, 10691 Stockholm, Sweden

23 Space Research Center of the Polish Academy of Sciences, Bartycka 18A, 00-716 Warsaw, Poland

24 University of Massachusetts, Astronomy Dept., 710 N. Pleasant St., LGRT-619E, Amherst, MA 01003-9305, USA

25 N. Copernicus Astronomical Center, Rabianska 8, 87-100 Torun, Poland

26 Experimental Physics Department, National University of Ireland, Maynooth, Co Kildare, Ireland

27 Netherlands Organization for Applied Scientific Research (TNO), Stieltjesweg, 2628 CK Delft, The Netherlands

28 Applied Physics Department, Delft University, Delft, The Netherlands

29 Northrop Grumman Aerospace Systems, 1 Space Park, Redondo Beach, CA 90278, USA

30 Centro de Astrobiología (INTA-CSIC), Ctra de Torrejón a Ajalvir, km 4, 28850 Torrejón de Ardoz, Madrid, Spain

31 Institut de Radioastronomie Millimetrique, IRAM, 300 rue de la Piscine, 38406 St Martin d'Hères, France

32 Osservatorio Astrofisico di Arcetri-INAF- Largo E. Fermi 5, 50100 Florence, Italy

33 European Space Astronomy Centre, ESA, PO Box 78, 28691 Villanueva de la Cañada, Madrid, Spain

34 European Organization for Astronomical research in the Southern Hemisphere, Karl-Scwarzschild-strasse 2, 85748 Garching, Germany

35 NASA Herschel Science Center, California Institute of Technology, Pasadena CA, USA 